\title{
Geología de la presa Ángel Cruz (Aija - Ancash)
}

\author{
Geoly of Angel Cruz dam (Aija, Ancash)
}

Pedro Hugo Tumialán De la Cruz'1, Eloy Maguiña Fernández²

\section{RESUMEN}

La presa Ángel Cruz (Aija - Ancash) se emplazará en el Cañón Mellizo del río Aija, a $2.5 \mathrm{~km}$ al N $85^{\circ} \mathrm{O}$ del borde oeste de la Ciudad de Aija. Será una presa de arco de concreto con convexidad al N $60^{\circ} \mathrm{E}$, emplazado en cuarcitas de la Formación Chimú del Cretáceo inferior de $246 \mathrm{~m}$ de altura. La parte superior de la presa tiene como cota 3,257.7 msnm y una longitud en su borde superior de $730 \mathrm{~m}$, represará 500 millones de metros cúbicos de agua del río Aija, formado por la intersección de los ríos La Merced, Santiago y Yachún. Las aguas que serán represadas por esta presa se usará para irrigación de terrenos áridos de la Costa de Huarmey y para el abastecimiento continuo de agua para cinco centrales hidroeléctricos en el río Aija. Debe considerarse la geodinámica externa e interna que afectará a la Presa y al agua represada. Considerar el impacto ambiental que ocasionará en la zona el represamiento de las aguas.

Palabras clave: Geología, presa, Ángel Cruz, Aija, Ancash

\section{ABSTRACT}

The Angel Cruz Dam (Aija - Ancash) will be located in the Mellizo Canyon of the Aija River at $2.5 \mathrm{~km} \mathrm{~N} 85^{\circ} \mathrm{O}$ west limit of the Aija town. It will be a Concret Arc Dam convexed to $\mathrm{N} 60^{\circ} \mathrm{E}$ in quartzite Chimú Formation of lower Cretaceos with $246 \mathrm{~m}$ high. The upper part of the Dam has an altitude of 3,257 msnm and a length of $730 \mathrm{~m}$. It will dammed 500 millon cubic meters of water of Aija river formed by the intersection of rivers La Merced, Santiago and Yachún. The water to be impounded by the Dam will be used for the irrigation of arid lands of the Coast of Huarmey and also for the continous supply of water for five Hidroelectric Central in the Aija river. External and internal geodynamics that will affect the dammed water and the Dam as well must be considered. It is also important to consider the inviromental impact that will result in the impoundment of water.

Key words: Geology, Dam, Angel Cruz, Aija, Ancash

1 Profesor de la Escuela de Ingeniería Geológica de la Facultad de Ingeniería Geológica, Minera Metalúrgica y Geográfica de la Universidad Nacional Mayor de San Marcos. E-mail: phtumialan@hotmail.com

2 Presidente del Proyecto: Presa Ángel Cruz (Aija - Ancash). E-mail: eloymagfernandez@gmail.com 


\section{GEOMORFOLOGÍA}

La presa Ángel Cruz (Aija - Ancash) se emplazará en el Cañón Mellizo del río Aija a $2.6 \mathrm{~km}$ al N 85 O del borde oeste de la ciudad de Aija.

Esta presa represará las aguas del río La Merced formado por sus tributarios en su flanco oeste por el río Yachún, cuya intersección está a $720 \mathrm{~m}$ al $\mathrm{N} 34^{\circ} \mathrm{E}$ de la presa; en su flanco este por el río Santiago cuya intersección está a $880 \mathrm{~m}$ al N $35^{\circ}$ E de la presa, el río Santiago está a $160 \mathrm{~m}$ al norte de la ciudad de Aija (Fig. 1). El río La Merced aguas debajo de su intersección con el río Yachún se denomina río Aija.

El río Aija en el tramo de la presa tiene un rumbo de $\mathrm{S} 30^{\circ} \mathrm{O}$ (Fig.1).

En el sector del emplazamiento de la presa conocida como Mellizo es angosto con paredes de gran pendiente de $30 \mathrm{~m}$ en su parte inferior tipo cañón; a mayor cota de este Cañón el valle tiene menor pendiente, significa un rejuvenecimiento del valle inicial por el levantamiento de la zona, una mayor erosión y formación del Cañón.

Los ríos referidos a cotas mayores de $3200 \mathrm{msnm}$ son valles fluviales de flancos amplios en forma de $\mathrm{V}$, sobre los 3800 msnm hubo erosión glaciar en el Cuaternario antiguo dando lugar a valles glacio-fluviales, los valles glaciares tiene una sección transversal en forma de "U".

La erosión actual del río Aija en el sector de la presa es intensa por su mayor velocidad porque el cañon es angosto sin presencia de terraza fluvial o aluvional y escaso suelo fluvial en el fondo del río en el sector de la presa.

\section{ESTRATIGRAFÍA}

La estratigrafía de la zona está representada por cuarcitas de la Formación del Chimú del Cretáceo inferior, con un espesor de $420 \mathrm{~m}$.

Posterior a la depositación de la Formación Chimú hubo una inmersión de la zona, en el fondo marino se depositaron calizas y lutitas de $50 \mathrm{~m}$ de espesor de la Formación Santa.

En el fondo marino sobre las calizas de la Formación Santa se depositaron lutitas de la Formación Carhuaz con un espesor de $1500 \mathrm{~m}$.

Las Formaciones Chimú, Santa y Carhuaz pertenecen al Grupo Goyllarisquizga del Cretáceo inferior (Figura N. ${ }^{\circ} 1$ ).

En la superficie de manera parcial se tiene áreas de suelo coluvial en las pendientes de los valles y suelo fluvial en el fondo del río, suelo fluvio aluvional en las terrazas de los ríos aguas arriba de la presa, esos suelos son del Cuaternario reciente.

\section{GEOLOGÍA ESTRUCTURAL}

La cuarcita Chimú forma un anticlinal asimétrico. El eje del anticlinal tiene un rumbo $\mathrm{N} 70^{\circ} \mathrm{O}$.

En el flanco NE de este anticlinal los estratos tienen un rumbo que varia de $\mathrm{N} 64^{\circ} \mathrm{O}$ a $\mathrm{N} 65^{\circ} \mathrm{O}$, un buzamiento de $84^{\circ} \mathrm{NE}$. A $260 \mathrm{~m}$ al $\mathrm{N} 30^{\circ}$ E del referido eje del anticlinal en el río Aija está el contacto de la cuarcita Chimú con la Formación Santa y más al $\mathrm{N} 30^{\circ}$ E la Formación Carhuaz, concordantes a la posición estructural de las cuarcitas.

El flanco SO del eje del anticlinal referido los estratos de cuarcitas tienen un rumbo $\mathrm{N} 70^{\circ} \mathrm{O}$, un buzamiento de $79^{\circ} \mathrm{SO}$. A $160 \mathrm{~m}$ del eje del anticlinal al S $40^{\circ} \mathrm{O}$ del río Aija está el contacto de las cuarcitas Chimú con la Formación Santa y más al S $40^{\circ}$ O la Formación Carhuaz concordantes a la posición estructural de las cuarcitas (Cobbing, J. et al. 1996).

\section{ASPECTOS GEOTÉCNICOS}

La presa será una presa de arco de concreto, tendrá una distancia vertical de $246 \mathrm{~m}$, una longitud de corona de 730 m, será una de las presas más altas en el Perú. Represará 500 millones de metros cúbicos de agua.

El ancho del río en la base de la sección de la presa es de $30 \mathrm{~m}$, por la fuerte corriente del agua del río Aija (al SO de la confluencia de los ríos La Merced, Yactún y Santiago el río La Merced se llama río Aija) no se ha depositado mucho material cuaternario en el fondo del río en la sección del emplazamiento de la presa por la fuerza de la corriente de agua. La parte convexa de la presa de Arco de Concreto se emplazará en cuarcitas Chimú a $40 \mathrm{~m}$ al S $30^{\circ} \mathrm{O}$ del contacto de la cuarcita Chimú con la caliza lutita Santa, su base y estribos estarán en las cuarcitas Chimú.

Las cuarcitas de la Formación Chimú son muy resistentes a los esfuerzos de compresión y no sufrirán ningún tipo de erosión por los agentes meteóricos como el agua, el oxígeno y cambios de temperatura.

Estructuralmente las cuarcitas Chimú están como estratos de espesores decimétricos, es decir por estratos anchos, la posición de estos estratos son de gran inclinación en el sector de la presa, de $84^{\circ}$ a $79^{\circ} \mathrm{NE}$ respecto a la horizontal, de rumbo $\mathrm{N} 60^{\circ} \mathrm{O}$, es perpendicular al esfuerzo que ejercerá el agua del embalse represado.

El esfuerzo horizontal del agua tendrá un rumbo a $\mathrm{S} 30^{\circ} \mathrm{O}$, su mayor intensidad estará a $1 / 3$ de la altura de la presa, es decir a $246 \mathrm{~m} / 3=82 \mathrm{~m}$ de la base del río. 
El esfuerzo horizontal se transmitirá a los estribos de la Presa que tienen excelentes condiciones geotécnicas a los esfuerzos que se aplicará sobre dicha cuarcita.

La presa de concreto será en forma de arco convexo hacia el $\mathrm{N} 30^{\circ} \mathrm{E}$. El espesor de la presa de concreto en la parte superior será menor respecto a su espesor en su base. Las dimensiones y los aspectos ingenieríles de la presa de arco los dará la Ingeniería Civil.

La base de la presa de arco de concreto se construirá en la cuarcita Chimú, para lo cual se determinará el espesor del material cuaternario en el fondo del río Aija que no será de mucho espesor. Este espesor inicialmente se podrá determinar con prospección geofísica de resistividad eléctrica o por el método de prospección geofísica sísmica, los cuales serán comprobados por excavaciones verticales. La presa de Concreto de Arco debe estar bien empotrado en la base y en los estribos para evitar problemas de deslizamientos, la profundidad de la base de la presa lo determinará la Ingeniería Civil.

Un aspecto sumamente importante en esta presa son las posibles filtraciones que podrían producirse por la base y por los dos estribos de la presa, además filtraciones por algún lugar del área total del agua represada considerado como el área del embalse. Si hubiera una falla distrital o regional que cruzará el área embalsada podría producirse fuga por dicha falla, no se observa una falla distrital o regional en el futuro embalse de agua represada.

Respecto a las posibles filtraciones en los estribos y en la base debe realizarse las pruebas para ver la capacidad de impermeabilidad de los estribos y de la base. La cuarcita es impermeable como roca, en los estribos y en la base podría producirse fracturas, fallas; además, las superficies de los estratos de la cuarcita son superficies por donde puede producirse percolación de agua. Para estudiar este aspecto se hará una malla de perforaciones de $20 \mathrm{~m}$ de longitud en cada perforación, en dichas perforaciones se inyectarán agua a una presión de 80 libras/pulgada cuadrada, si el agua fluyera por alguna de las perforaciones será un sector permeable.

En la malla de perforaciones, la distancia entre cada perforación será de $4 \mathrm{~m}$. La posición de las perforaciones será perpendicular al perfil de los estribos y verticales en la base horizontal de la presa. Si la base de la presa tuviera más de $4 \mathrm{~m}$ de ancho se tendrá otras filas de perforaciones separados entre filas $4 \mathrm{~m}$, sucederá algo similar para los estribos si su ancho fuera de más de $4 \mathrm{~m}$.

Los sectores donde hubiera fuga de agua en las perforaciones serán zonas permeables; en esas perforaciones se inyectará lechada de cemento, el cual se filtrará en las fracturas en las superficies de los estratos de la cuarcita, la lechada de cemento al fraguar dará a esos sectores una condición impermeable.

Es probable, si la condición geotécnica lo exigiera, que se tenga que perforar túneles horizontales en cada estribo, unos $20 \mathrm{~m}$ de longitud horizontal en cada túnel. En cada túnel cada $4 \mathrm{~m}$ realizar perforaciones radiales al túnel $20 \mathrm{~m}$ de longitud en el piso, en el techo y en las dos paredes del túnel; estas perforaciones radiales estarán en un plano perpendicular al eje horizontal del túnel, en cada perforación realizar la prueba de impermeabilidad, e inyección de lechada de cemento, si lo necesitara. En $246 \mathrm{~m}$ verticales se hará 6 niveles de túneles a las cotas 3051.7, 3091.7, 3131.7, 3171.7, 3211.7, 3251.7 msnm, túneles en cada estribo de la presa. Con un total de 6 túneles en cada estribo, 12 túneles en los 2 estribos. Si cada túnel tiene $20 \mathrm{~m}$, tendremos $240 \mathrm{~m}$ de túneles.

Si fuera una sola línea de perforación de $20 \mathrm{~m}$, perpendicular al perfil de los estribos y de la base de la presa, se tiene aproximadamente en cada estribo $246 \mathrm{~m} / 4=61.5$ perforaciones consideremos 62 perforaciones, en los 2 estribos $62 \times 2=124$ perforaciones, en la base de $30 \mathrm{~m}$ de ancho se tendrá $30 / 4=7.5$ perforaciones, consideremos 8 perforaciones. En total se tendrá 132 perforaciones. Si cada perforación tiene $20 \mathrm{~m}$ de longitud, el total de perforaciones será de $132 \times 20=2640 \mathrm{~m}$ de perforación. Si el ancho de la presa fuera mayor a los $4 \mathrm{~m}$, el metraje de perforaciones será varias veces mayor a lo indicado.

El costo de este trabajo es un gran porcentaje del costo de la presa.

Durante el funcionamiento de la presa debe estudiarse la cantidad en metros cúbicos de lodo que llevan los ríos durante un año a la presa y la cantidad en metros cúbicos de lodo que sale de la presa durante un año, hallando la diferencia de la cantidad de lodo que entra y que sale de la presa tendremos la cantidad de lodo en metros cúbicos que se quedará en la presa durante un año. Dividiendo el volumen de agua represada por la presa entre la cantidad de lodo en metros cúbicos que queda en la presa durante un año tendremos la vida de la presa.

Para la construcción de la presa se desviará el curso del río, el cual se hará por un túnel por cualquiera de los estribos. Dicho desvío se hará antes de la presa, digamos $50 \mathrm{~m}$ antes de la presa, en su inicio el túnel de desvío atravesará calizas y lutitas de la Formación Santa, el túnel en las calizas no tendrá problemas de estabilidad por cuanto el túnel atravesará estratos perpendiculares a la dirección del túnel, si hubiera horizontes de lutita habrá problemas de estabilidad en el túnel el cual se solucionará con shotcrete y arcos de acero, la mayor longitud estará en las cuarcitas Chimú sin problemas. 
En el sector final del túnel de desvío estará en cuarcita Chimú, se adecuará una cámara donde funcionará la Primera Estación Hidroeléctrica. Esta cámara en la cuarcita Chimú no tendrá ningún problema geotécnico respecto a su estabilidad porque la cuarcita es una roca muy dura y estable.

\section{GEOLOGÍA DEL MEDIO AMBIENTE}

Las aguas represadas, además de ser aprovechadas para generar electricidad en cinco centrales hidroeléctricas en el río Aija, se emplearán para regadío de terrenos secos en la costa en Huarmey y terrenos de cultivo aguas abajo de la presa, por lo tanto debe cuidarse la acidez del agua, pues perjudicará a su utilización aguas abajo de la presa, la acidez del agua debe ser neutra $\mathrm{pH}$ 7, o ligeramente básico mayor de $\mathrm{pH} 7$.

El río Santiago es temporal, pasa cerca a Aija, está más contaminado con aguas ácidas por la explotación de las diferentes estructuras mineralizadas polimetálicas (vetas, cuerpos) que se encuentran en su cuenca, entre ellas las minas de Hércules, Coturcán, Huancapetí, La Conquista, Washington, San Hilarión, La Recuperada, Santa Cruz, Sarita I y Siempre Rosita.

La cuenca del río La Merced tiene los yacimientos y prospectos Pilar, Estrella, Boreal, Cinco Hermanos, Félix III, Félix IV.

La cuenca del río Yactún viene desde la zona de Huinac, donde se explora a escala de pequeña minería los yacimientos y prospectos Andes Tres, Cerro Huinac. Este río contribuirá en la contaminación del área represada (Tumialán, P.H. 2003).

Por lo tanto la cuenca del río Santiago producirá una mayor contaminación, seguido en importancia de contaminación la cuenca del río Yactún que viene desde la zona mineralizada de Huínac, en tercer orden de contaminación es producido por la cuenca del río La Merced.

En las tres cuencas no se tiene plantas concentradoras que sería de un problema mayor, solo se observa el lavado y oxidación de los afloramientos de las estructuras mineralizadas, del agua ácida que drenan sus labores mineras y sobre todo la oxidación y lavado de sus canchas (ruma de minerales extraídos y depositados cerca de las bocaminas). Una de la remediaciones para disminuir la contaminación es colocar calizas trituradas (tamaño de un puño cerrado) en las bocaminas (Maguiña, E. 2008). Deberá medirse las concentraciones de los elementos metálicos en ppm y en ppb y determinar si esas concentraciones están en los rangos permisibles para no contaminar las aguas de la presa.

Por este problema de contaminación no hay peces en los tres ríos, inclusive han desaparecido los sapos.

\section{GEODINÁMICA EXTERNA E INTERNA QUE AFECTARÁN LA ECOLOGÍA DE LA ZONA}

La laguna que se creará al represar el agua por la presa cambiará el microclima en los pueblos cercanos al borde de la Presa como en Aija, La Merced y caseríos. Este cambio del microclima afectará el ecosistema con relación al reino animal, sobre todo a las aves y a la vegetación cerca al borde de la laguna represada.

Como geodinámica externa por el exceso de lluvias se tendrá deslizamientos, desprendimientos del suelo cercano al borde de los ríos, con algunos problemas de asentamientos en los suelos, con consiguiente acarreo de arcilla, limo, arena, gravilla, grava, cantos angulosos y cantos rodados al fondo de la laguna futura que se creará por el represamiento, con lo cual le restará paulatinamente el volumen de agua represada.

El Perú ha sido es y será un país con ocurrencia de sismos. En esta zona, uno de los sismos fuertes registrados fue el sismo ocurrido el 31 de mayo de 1970 de grado VII de la Escala de Richter, con muchos problemas. Por el tipo de roca y su posición estructural, la roca sobre la cual se construirá la presa es resistente a futuros sismos.

Un problema importante para la presa es el estudio hidrológico (régimen de lluvias, caudales de los ríos) e hidrogeológico (aguas subterráneas). Se debe construir artesanalmente con rocas y arcillas en las partes altas de las 3 cuencas un número muy grande de pequeñas presas, las cuales almacenarán agua en invierno, en las épocas de verano sin lluvias, las aguas de estas minipresas aportarán agua que percolarán por los suelos y fracturas dando un aporte adicional de agua a la laguna represada por la presa en verano.

Una vez construida la presa, aguas abajo de esta los manantiales existentes pueden tener más caudal de agua o pueden aparecer otros manantiales, por cuanto desconocemos las interconexiones de pequeñas fracturas o fallas que llevarán aguas abajo parte del agua represada que no se ven.

Se debe construir una estación metereológica que dará datos metereológicos para la presa (temperatura, dirección y velocidad de viento, humedad, régimen de lluvia).

Se hará un levantamiento topográfico del fondo de la presa y del fondo del embalse cada tres años, con medidas de la profundidad del agua embalsada con un bote ubicado con GPS, para determinar el suelo que fue transportado a la presa y que no ha sido evacuado.

Esta obra es de mucha trascendencia, tanto en la Energía Eléctrica que se generará como en el regadío de áreas secas en la Costa (parte de las áreas de 
Haurmey), no solo merece el apoyo de gobiernos extranjeros como Alemania, sino el apoyo del gobierno peruano por parte del Ministerio de Energía y Minas y por el Ministerio de Agricultura.

El esfuerzo inicial que están realizando las personas que nacieron en Aíja o son descendientes de personas que nacieron en esa provincia tiene mucho valor $\mathrm{y}$ merece todo apoyo. Sea este trabajo un grano de apoyo al gran esfuerzo que requiere esta obra.

\section{REFERENCIAS}

1. Cobbing, J. et al. 1996. Geología de los Cuadrángulos de Huaraz, Recuay, La Unión, Chiquian y Yanahuanca.. INGEMMET, Boletín N. ${ }^{\circ} 76$, pág. 35-143. 1996.

2. Maguiña, Eloy. Comunicación personal, 2008.

3. Tumialán, Pedro Hugo. Compendio de Yacimientos Minerales del Perú. INGEMMET, Boletín N ${ }^{\circ}$ 10 de la serie B, pág. 290-303. 2003.
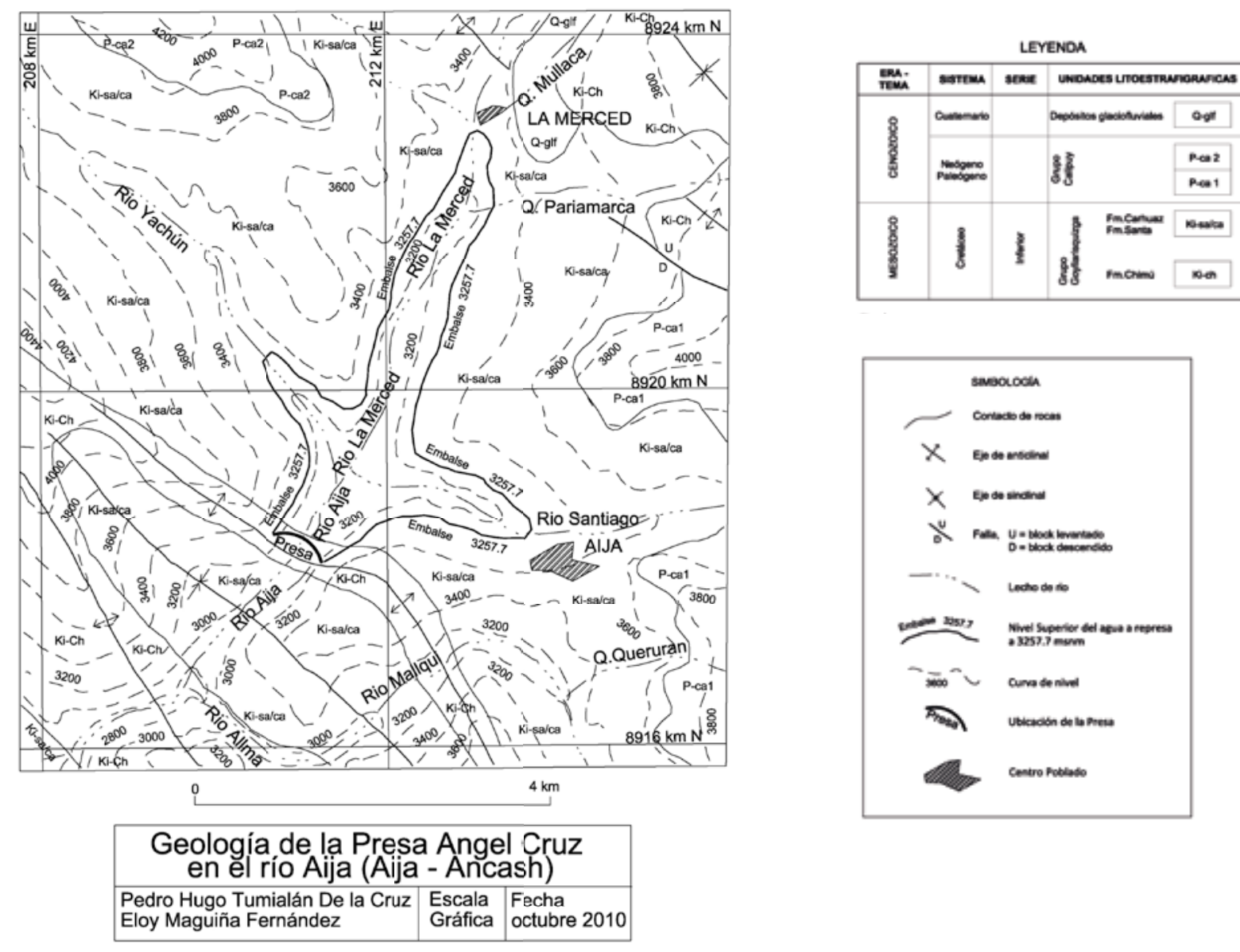

Figura N. ${ }^{\circ}$ 1. Geología de la presa Ángel cruz en el río Aija (aija - Ancash). 